This is an electronic reprint of the original article. This reprint may differ from the original in pagination and typographic detail.

Author(s): Annala, Riia; Suhonen, Aku; Laakkonen, Heikki; Permi, Perttu; Nissinen, Maija

Title: $\quad$ Structural Tuning and Conformational Stability of Aromatic Oligoamide Foldamers

Year: $\quad 2017$

Version:

Please cite the original version:

Annala, R., Suhonen, A., Laakkonen, H., Permi, P., \& Nissinen, M. (2017). Structural Tuning and Conformational Stability of Aromatic Oligoamide Foldamers. Chemistry: A European Journal, 23(65), 16671-16680. https://doi.org/10.1002/chem.201703985

All material supplied via JYX is protected by copyright and other intellectual property rights, and duplication or sale of all or part of any of the repository collections is not permitted, except that material may be duplicated by you for your research use or educational purposes in electronic or print form. You must obtain permission for any other use. Electronic or print copies may not be offered, whether for sale or otherwise to anyone who is not an authorised user. 


\title{
Structural tuning and conformational stability of aromatic oligoamide foldamers
}

\author{
Riia Annala,${ }^{[a]}$ Aku Suhonen, ${ }^{[a]}$ Heikki Laakkonen, ${ }^{[a]}$ Perttu Permi ${ }^{[a, b]}$ and Maija Nissinen ${ }^{*[a]}$
}

\begin{abstract}
A series of aromatic oligoamide foldamers with two or three pyridine-2,6-dicarboxamide units as their main folding motifs and varying aromatic building blocks as linkers have been synthetized to study the effects of the structural variation on the folding properties and conformational stability. Crystallographic studies showed that in the solid state the central linker unit either elongates the helices and more open S-shaped conformations, compresses the helices to more compact conformations or acts as a rigid spacer separating the pyridine-2,6-dicarboxamide units, which for their part add the predictability of the conformational properties. Multidimensional NMR studies showed that, even in solution, foldamers show conformational stability and folded conformations comparable to the solid state structures.
\end{abstract}

\section{Introduction}

During the last decades our understanding about biological processes, such as the catalytic activity and selectivity of enzymes, has greatly increased. This understanding has brought numerous new opportunities for chemists to learn from and adapt towards chemical applications. Synthetic biomimetic oligomers known as foldamers aim to combine the advantages of biological polymers to the favourable properties of synthetic oligomers, such as endurance of varying temperatures, $\mathrm{pH}$ and salt concentrations, and possibility to function in organic solvents. $^{[1,2]}$ Their structural rigidity obtained by, for example, repeating aromatic moieties connected by amide or urea bonds allows smaller size and a simpler design of the molecules and adds predictability and stability to their folding and secondary structures, which is the basis for potential applications of foldamers. ${ }^{[3]}$

A number of different types of aromatic oligoamides have been studied both in solution and in the solid state giving indication on how structural features and interactions affect the folding and conformational properties. ${ }^{[4]}$ In solution the folding properties are greatly affected by competitive interactions with solvent, thus diminishing the predictability of the folding in solution. ${ }^{[5]}$ In the

[a] R. Annala, Dr. Aku Suhonen, H. Laakkonen, Prof. Dr. M. Nissinen Department of Chemistry, Nanoscience Center University of Jyvaskyla P.O. Box 35

40014 University of Jyvaskyla (Finland)

E-mail: maija.nissinen@jyu.fi

[b] Prof. Dr. P. Permi

Department of Chemistry and Department of Biological and Environmental Sciences, Nanoscience Center

University of Jyvaskyla

P.O. Box 35

40014 University of Jyvaskyla (Finland)

Supporting information for this article is available on the WWW under http:// solid state, on the other hand, the requirement of the closest packing and possibility of small molecule inclusion either in the interstice between the foldamers or inside the fold, may alter the folding and conformational properties. ${ }^{[6,7]}$ The properties of aromatic foldamers, such as the flexibility ${ }^{[8]}$, water solubility ${ }^{[9]}$ and overall conformation ${ }^{[10]}$ as well as diameter ${ }^{[11]}$ and chirality ${ }^{[12]}$ of the helix, have been tuned by the addition of different types of monomers. The most common trend has been the addition of aliphatic monomers to make heterogeneous foldamers ${ }^{[13,14]}$ but also foldamers with different aromatic sequences have been made to create, for example, foldamer capsules ${ }^{[15]}$ and selective receptors $^{[16]}$.

The pyridine-2,6-dicarboxamide unit is one of the structural motifs used as a turn unit to impose helical conformations on oligomers. ${ }^{[17]}$ Our previous studies with a series of aromatic oligoamides (4-5 aromatic rings) with a pyridine-2,6dicarboxamide center have shown that this type of short foldamers reliably adopt two, almost equally stabile folded conformers with only small variances in their hydrogen bonding and structural features. ${ }^{[18-20]}$ Which conformer, denoted as @ or $S$ according to their overall shape (Scheme 1), ${ }^{[1]}$ prevails depends on the chemical structure of the foldamer as well as environment, such as crystallization conditions and solvent. The conformers and folding of these molecules are based on intramolecular hydrogen bonding between the amide groups and the pyridine-2,6-dicarboxamide unit, which forms a suitable niche for multiple interactions, whereas aromatic interactions seem to play a minor role in the folding preferences of the oligoamides. Interestingly, the @ conformer with three intramolecular hydrogen bonds forming to a single carbonyl oxygen, resembles closely an oxyanion hole motif found in the active sites of certain enzymes. ${ }^{[22]}$ Artificial, non-peptidic models for oxyanion hole are not common, as only a few examples of amide and ester carbonyls motifs as acceptors for multiple hydrogen bonds have been described. ${ }^{[23]}$ Thus, aromatic oligoamide foldamers possess great potential as structural and functional mimics of enzyme catalysts.

In our current study, we show that the oxyanion hole motif and the folding patterns are preserved, when the size of the foldamer increases and the number of pyridine-2,6-dicarboxamide units is doubled or tripled (Scheme 1). The spacer unit separating the pyridine-2,6-dicarboxamide units affects the overall folding of the foldamer by preventing certain geometries and/or inducing helicity or compact conformations by participating in intramolecular hydrogen bonding. This indicates that conformational adaptability of foldamers can be controlled with suitable spacers without losing the essential folding motifs and potential binding sites, such as oxyanion hole motif.

\section{Results and Discussion}

A series of five oligoamide foldamers (7-9 aromatic rings) were synthetized applying the procedures described in our previous 
papers ${ }^{18-20}$ (see SI for details). The conformational features and the stability of the fold and oxyanion hole motif were studied in the solid state by X-ray crystallography and compared with the solution state information obtained by multidimensional NMR spectroscopy. In foldamers 1-3 two pyridine-2,6-dicarboxamide units are separated by 1-3 ortho-substituted phenyl rings and 0 2 amide bonds, whereas in foldamers 4 and $\mathbf{5}$ either a third pyridine-2,6-dicarboxamide center or its phenyl analogue are used as a spacer. The ortho substituted phenyl rings of foldamers 1-3, especially three consequent rings, act as a linear type of spacer, whereas meta substituted centers of 4 and 5 automatically cause a different type of overall fold, which in the case of the pyridine-2,6-dicarboxamide center of $\mathbf{5}$ leads to a helical fold stabilized by intramolecular hydrogen bonding to pyridine.

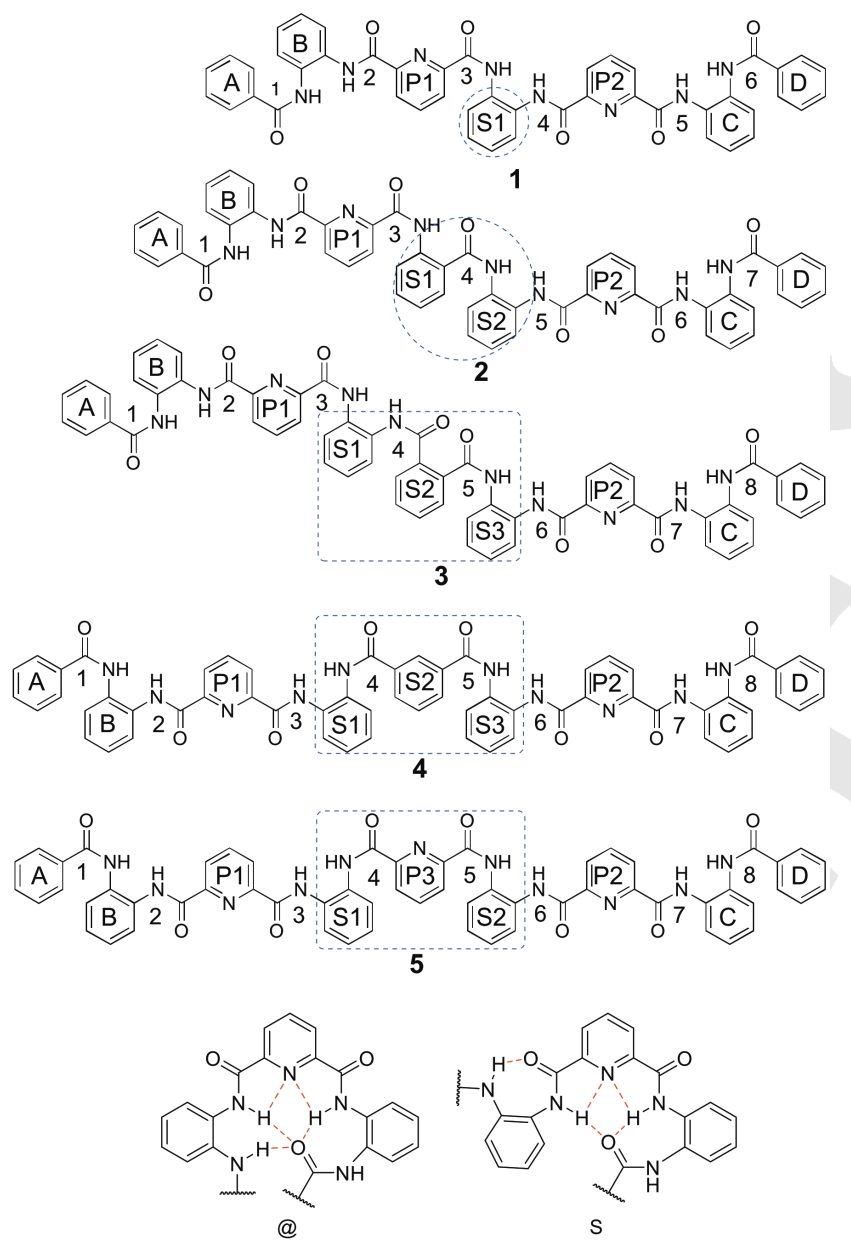

Scheme 1. Chemical structures of the foldamers 1-5 including notifications of benzene and pyridine rings and numbering of $\mathrm{C}=\mathrm{O}$ and $\mathrm{NH}$ groups. The spacer groups are circled with dotted lines. A schematic presentation of two observed folding patterns around pyridine-2,6-dicarboxamide center (bottom row).

\section{Solid state conformations}

The crystallization studies of the foldamers resulted in 17 different crystal structures. All of the crystal structures obtained for 1-5 were solvates, which indicates that folded molecules cannot pack very efficiently due to their complex and awkward shape. In the structures of foldamers 1-3 and 5 at least one of the pyridine-2,6-carboxamide units adopts the predicted folded @ conformation (Scheme 1; Table 1; Table S-3 in SI) by 2-3 hydrogen bonds from adjacent $\mathrm{C}=\mathrm{O}$ group to $\mathrm{NH}$ groups next to pyridine ring, thus retaining the desired oxyanion hole motif. Foldamer $\mathbf{4}$ is the only exception to this as both pyridine-2,6dicarboxamide centers are in a more open $\mathrm{S}$ conformation.

Table 1. 17 solvate structures of the foldamers 1-5 and the conformations of the pyridine centers

\begin{tabular}{|c|c|c|c|}
\hline Structure & Center P1 & Center P2 & Notes \\
\hline $\begin{array}{l}\text { 1-DMA- } \mathrm{H}_{2} \mathrm{O} \\
\text { 1-MeCN- } \mathrm{H}_{2} \mathrm{O}\end{array}$ & $@$ & $@$ & isomorphous \\
\hline $\begin{array}{l}\text { 1-DMF- } \mathrm{H}_{2} \mathrm{O} \\
1-\mathrm{MeOH} \\
1-\mathrm{DMSO} \\
1-\mathrm{DCM}\end{array}$ & 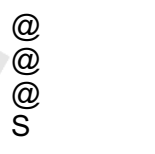 & $\begin{array}{l}@ \\
@ \\
@\end{array}$ & \\
\hline $\begin{array}{l}\text { 2-MeCN } \\
\text { 2-EtOAc }\end{array}$ & $@$ & $S$ & isomorphous \\
\hline $\begin{array}{l}\text { 2-DCM } \\
\text { 2-DMSO } \\
\text { 3-DMA } \\
\text { 3-DMSO } \\
\text { 4-EtOAC }\end{array}$ & $\begin{array}{l}\stackrel{@}{@} \\
@ \\
@ \\
\mathrm{~S}\end{array}$ & $\begin{array}{l}\mathrm{S} \\
\mathrm{S} \\
@ \\
@ \\
\mathrm{~S}\end{array}$ & $\begin{array}{l}\text { trans } \\
\text { cis }\end{array}$ \\
\hline $\begin{array}{l}\text { 5-Ac } \\
5-\mathrm{DCM} \\
5-\mathrm{DMF}\end{array}$ & $@$ & $@$ & isomorphous \\
\hline 5- $\mathrm{CHCl}_{3}$ & $\mathrm{~S}$ & @ & \\
\hline
\end{tabular}

\section{Foldamer 1}

Altogether six single crystal structures of the foldamer 1 were obtained (see SI for crystallization details), but only two variations of overall conformation were observed. The isomorphous structures of $1-\mathrm{DMA}-\mathrm{H}_{2} \mathrm{O}$ and $1-\mathrm{MeCN}-\mathrm{H}_{2} \mathrm{O}$ adopt an overall tight helical conformation with both pyridine centers in an @ fold (@/@; Figure 1). The conformations of 1-DMSO, 1$\mathrm{MeOH}$ and 1-DMF- $\mathrm{H}_{2} \mathrm{O}$ solvates are also similar with both pyridine centers in @ fold (see ESI Figure S-1 for an overlay of all @/@ structures). In these structures either one (1-MeOH) or both (1-DMA- $\mathrm{H}_{2} \mathrm{O}, 1-\mathrm{MeCN}-\mathrm{H}_{2} \mathrm{O}, 1-\mathrm{DMF}-\mathrm{H}_{2} \mathrm{O}$ and 1-DMSO) of the outmost phenyl rings have a $\mathrm{CH}-\pi$ interaction with the spacer phenyl ring. 1-DCM solvate has a less folded structure, as one of the pyridine-2,6-dicarboxamide centers adopts an open $\mathrm{S}$ fold whereas the other one is in an @ fold (@/S; Figure 1). The outmost phenyl ring of the @ folded part of the molecule interacts with the spacer phenyl ring via $\mathrm{CH}-\pi$ interaction (3.041 A) like in the other structures.

The crystal packing in all structures of foldamer $\mathbf{1}$ is based on intermolecular hydrogen bonding. In isomorphous @/@- 
structures (1-DMA- $\mathrm{H}_{2} \mathrm{O}$ and $1-\mathrm{MeCN}-\mathrm{H}_{2} \mathrm{O}$ ) and in 1-DMF- $\mathrm{H}_{2} \mathrm{O}$ structure two water molecules connect two foldamers into pairs via bifurcated hydrogen bonds $\left(\mathrm{OH}_{\mathrm{w}} \ldots \mathrm{O}=\mathrm{C}\right.$; graph set $\left.R_{4}^{4}(20)\right)$ and further to chains formed by these pairs $\left(\mathrm{NH} 1 \ldots \mathrm{O}_{\mathrm{w}}\right.$, graph sets $C_{2}^{2}(13)$ and $R_{4}^{4}(20)$; Figure 1 ). The pairing is enhanced by $\pi-$ $\pi$ stacking between the pyridine centers $P 1$ of the adjacent molecules. The solvent (DMA, MeCN or DMF) is hydrogen bonded to the outer groove of the fold (NH6...Solvent; $\mathrm{D}(2)$ motif). The difference of the crystal structures of isomorphous structures and 1-DMF- $\mathrm{H}_{2} \mathrm{O}$ comes from the efficiency of packing. In 1-DMA- $\mathrm{H}_{2} \mathrm{O}$ and 1- $\mathrm{MeCN}-\mathrm{H}_{2} \mathrm{O}$ there is $\pi-\pi$ stacking between the end phenyl rings (D), which is prevented in 1-DMF- $\mathrm{H}_{2} \mathrm{O}$ structure by the inclusion of one extra solvent molecule between the rings (See SI Figure S-2). In 1-DMSO solvate the intermolecular hydrogen bonds orient to two DMSOs, which fill the interstice between the foldamers. The foldamers still pack into layers: the grooves of the folds assemble parallel to each other and interact via $\pi-\pi$ interactions (See SI; Figure S-2).
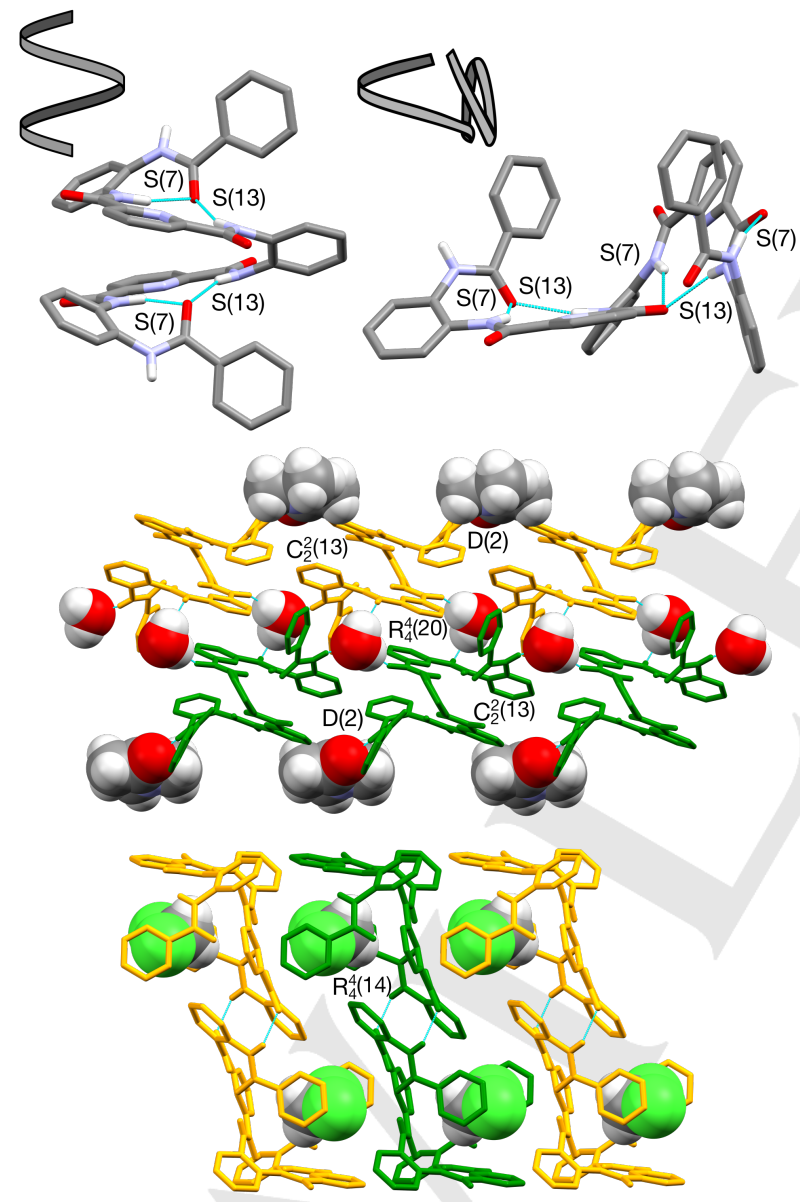

Figure 1 The conformations and schematic presentations of folding of 1-DMA $\mathrm{H}_{2} \mathrm{O}$ (top left) and 1-DCM (top right). Crystal packing of 1-DMA- $\mathrm{H}_{2} \mathrm{O}$ (middle) and 1-DCM structures (below) showing the inclusion of solvents and intermolecular hydrogen bonding motifs (turquoise lines and graph set notifications). Nonbonding hydrogens and disorder have been omitted for clarity and solvents are shown with space fill model.
In $1-\mathrm{DCM}$ and $1-\mathrm{MeOH}$ solvates there are direct hydrogen bonds between the foldamers as two intermolecular hydrogen bonds between $\mathrm{NH} 1$ and $\mathrm{C}=\mathrm{O} 2$ groups $\left(R_{4}^{4}(14)\right.$ motif) connect the foldamers into pairs. In case of the more open @/S conformation of 1-DCM structure, this leads to a niche for a disordered solvent molecule inside the pair of the awkwardly shaped molecules (Figure 1). In 1-MeOH solvate the pairs are further connected to chains via solvent mediated hydrogen bonds from $\mathrm{MeOH}$ to $\mathrm{NH} 6$ of one foldamer and $\mathrm{C}=\mathrm{O} 4$ of the next pair (see SI Figure S-2).
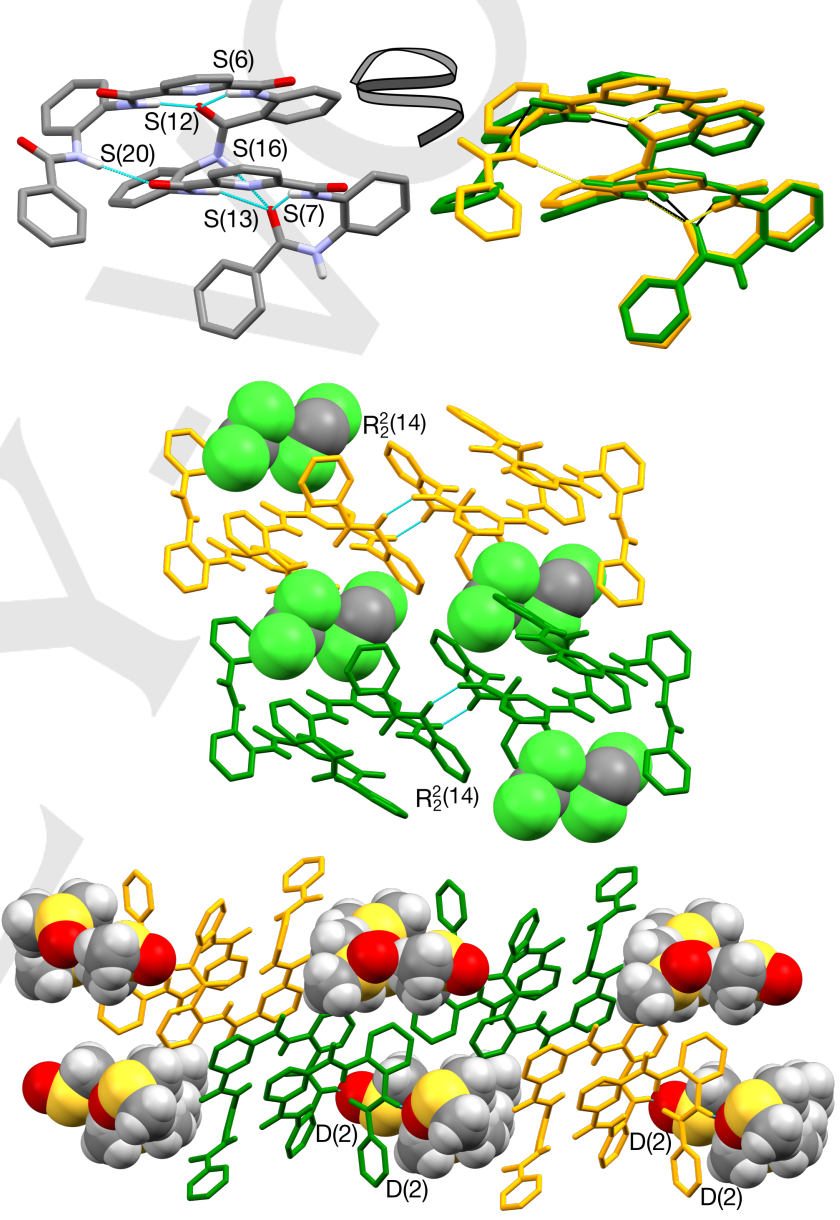

Figure 2 The conformations of 2-MeCN (top left) and an overlay presentation of the structures of 2-DMSO (orange) and 2-DCM (green; top right). The crystal packing of 2-DCM (middle) and 2-DMSO (below). Solvents are shown with space filling model and hydrogen bonds with turquoise bonds. Nonbonding hydrogens and disorder have been omitted for clarity.

\section{Foldamer 2}

Four different single crystal structures of foldamer 2 were obtained from acetonitrile, ethyl acetate, dichloromethane and DMSO solutions. Two of these structures, the MeCN and the EtOAc solvates (2-MeCN and 2-EtOAc), are isomorphous and the differences between all four structures are minor. In all four structures the P2 pyridine-2,6-dicarboxamide center adopts an @-fold, whereas the P1 center is in S-fold (Figure 3). The overall 
conformation is compact, almost helical structure where two pyridines have parallel displaced $\pi$-interactions with each other and the end of the S-fold surrounds the helical part. The similarities are facilitated by the unsymmetrical linker unit, where the $\mathrm{C}=\mathrm{O} 4$ group prefers to form the $\mathrm{S}$-fold by hydrogen bonds to the $\mathrm{NH}$ groups of the pyridine-2,6-dicarboxamide unit $\mathrm{P} 1$ (graph set motifs $\mathrm{S}(6)$ and $\mathrm{S}(12)$ ). The outmost $\mathrm{NH} 1$ finishes the S-fold by a hydrogen bond to $\mathrm{O} 2$ (S(7) motif; 2-DCM) or to $\mathrm{O} 5$ of the pyridine-2,6-dicarboxamide center P2 (S(20) motif; other solvates), which leads to slight difference in the orientation of the outmost phenyl ring. The @ folds around pyridine center P2 in isomorphous 2-MeCN and 2-EtOAc structures and in 2-DCM solvate are based on typical three hydrogen bonds between the outmost $\mathrm{C}=\mathrm{O}$ group $(\mathrm{O} 7)$ and $\mathrm{NH}$ groups of the $\mathrm{P} 2$ unit $(\mathrm{S}(7)$ and $\mathrm{S}(13)$ motifs) and to the next $\mathrm{NH}$ group $(\mathrm{NH} 4)$ in the linker $(\mathrm{S}(16)$ motif). In 2-DMSO, however, the third hydrogen bond is missing as the amide $\mathrm{NH} 4$ is hydrogen bonded to solvent $(\mathrm{D}(2)$ motif). Also in the case of foldamer 2 the crystal packing is based on the pairing of molecules via direct intermolecular hydrogen bonding (NH7...O6; $R_{2}^{2}(14)$ motif; Figure 2). The solvents are located inside the cleft formed by two hydrogen bonded pairs of foldamers (DCM) or interstice in the crystal lattice (EtOAc and $\mathrm{MeCN}$ ) (See SI Figure S-4). The exception to pairwise hydrogen bonding is the DMSO solvate, as DMSO disrupts this pattern and the intermolecular hydrogen bonds to two DMSO molecules (from $\mathrm{NH} 7$ and $\mathrm{NH} 4$ to $\mathrm{O} 10 \mathrm{~A}$ and $\mathrm{O} 30 \mathrm{~A} ; \mathrm{D}(2)$ motifs).
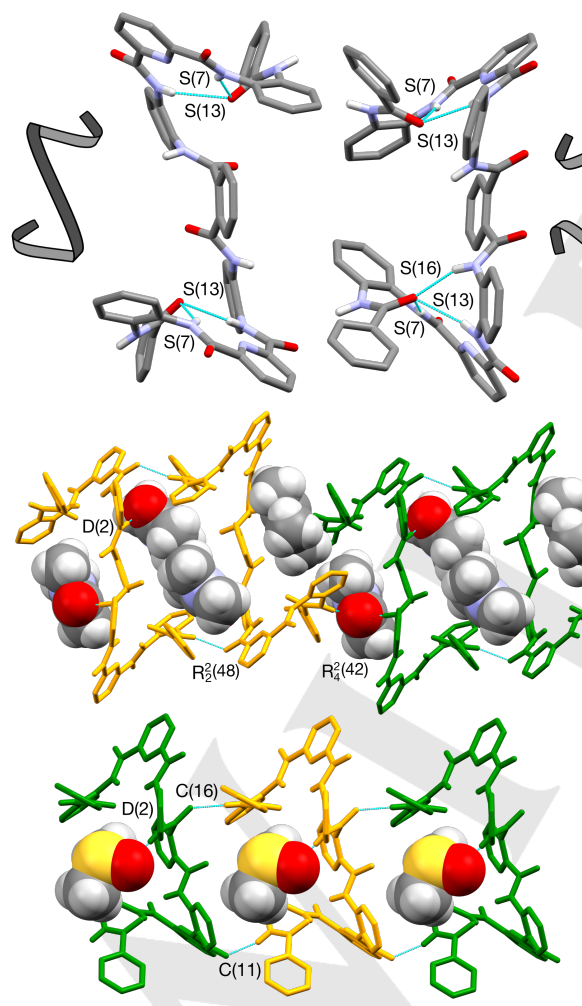

Figure 3 The solid state conformations and crystal packing of 3-DMA (top left and middle) and 3-DMSO (top right and below) showing the trans and cis orientations of pyridine-2,6-dicarboxamide centers in respect to spacer unit. Non-bonding hydrogens and disorder have been omitted for clarity and the solvents are shown with space fill model.

\section{Foldamer 3}

Two single crystal structures of the foldamer 3 were obtained from crystallizations in DMA and DMSO solution. Both structures have two @-folded pyridine-2,6-dicarboxamide units with typical hydrogen bonding patterns $(S(7)$ and $S(13)$ motifs), but the foldamer 3 does not have an overall helical conformation because the linker group constituting of three ortho-substituted phenyl rings is fairly rigid, thus separating the ends of the foldamer as independent folds, which can orient differently in respect to the spacer unit. The two conformers observed in the crystal structures are indeed distinctly different (Figure 3). In 3DMSO solvate the pyridine-2,6-dicarboxamide units of the molecule turn on the same side of the spacer (cis) giving a foldamer a bowl-like overall conformation with a solvent accessible cavity occupied by a disordered DMSO which is hydrogen bonded to $\mathrm{NH} 4$. The foldamer molecules are connected head-to-tail manner into continuous chains (NH8...O6; C(11) motif and $\mathrm{NH} 1 \ldots \mathrm{O} 4 ; \mathrm{C}(16)$ motif; Figure 3). In 3-DMA solvate the pyridine-2,6-dicarboxamide units are oriented on the different sides of the linker group (trans), which leaves the center of the foldamer open for interaction with solvents and enables the packing into ladder-like chains via direct intermolecular hydrogen bonding (O3...HN8) on one side and via solvent mediated hydrogen bonds on the other side $\left(\mathrm{NH} 1 \ldots \mathrm{O}_{\mathrm{s}} \ldots \mathrm{HN5}\right.$; Figure 5). Two DMA molecules are nested in between the directly hydrogen bonded foldamer pair and have intermolecular hydrogen bonds to the $\mathrm{NH} 4$ of the foldamers $\left(\mathrm{NH} 4 \ldots \mathrm{O}_{\mathrm{s}}\right)$. The conformational difference between the two solvates may be caused by the efficiency of packing, as nearly planar DMA enables denser packing than DMSO, as well as the hydrogen bonding preferences in each case.

\section{Foldamer 4}

The only crystal structure of foldamer $\mathbf{4}$ was obtained from ethyl acetate. The conformation is symmetrical with two $S$ folds oriented on the opposite sides of the central phenyl ring. The meta-substituted spacer of foldamer $\mathbf{4}$ is not as planar and rigid as the ortho-substituted analogue of the foldamer 3 , which might be a reason for the preference of $S$ folds. The conformation is stabilized to a compact entity by an additional intramolecular hydrogen bond from outmost carbonyl $\mathrm{C}=\mathrm{O}(\mathrm{O} 1$ and $\mathrm{O} 8)$ to central $\mathrm{NH}$ groups ( $\mathrm{NH} 5$ and $\mathrm{NH} 4$, respectively). The packing of 4-EtOAc is based on continuous chains formed by intermolecular hydrogen bond from $\mathrm{O} 3$ and $\mathrm{O} 6$ to $\mathrm{NH} 4$ and $\mathrm{NH} 5$ of the neighboring foldamers. Two disordered EtOAc molecules fill the interstice between the chains.

\section{Foldamer 5}

Four single crystal structures of the foldamer 5 were obtained, three of which (5-Ac, 5-DCM and 5-DMF) are isomorphous and nearly helical structures with the outmost pyridine-2,6dicarboxamide units adopting an @ fold. In this case, the fold is stabilized by only two hydrogen bonds (S(7) and $S(13)$ motifs) as additional hydrogen bonds are prevented because of hydrogen bonds to the pyridine-2,6-dicarboxamide center P3 at the spacer unit. This center facilitates significantly helical-type of folding by forming a third center for intramolecular hydrogen 
bonds. The helical folding of the foldamer leaves several hydrogen bonding groups exposed at the outer surface of the fold, which enables the formation of a complex intermolecular hydrogen bond network. The foldamers form pairs with two hydrogen bonds (NH8...O7) and these pairs are further connected to the chains of pairs via hydrogen bonds $(\mathrm{NH} 1 \ldots \mathrm{O} 6)$ which connects each foldamer to three other foldamers; to one with two hydrogen bonds, to two with one hydrogen bond.

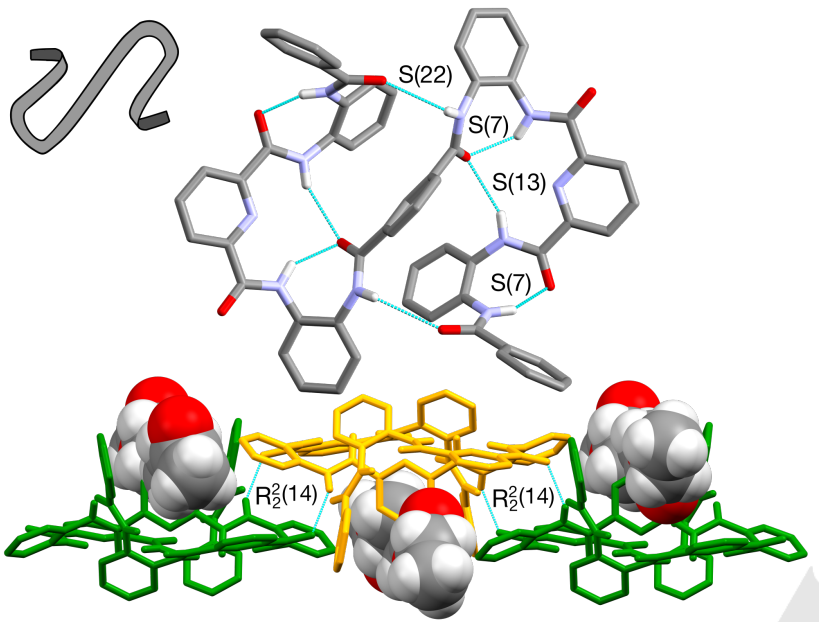

Figure 4 Conformation and crystal packing of 4-EtOAc. The conformation of the foldamer $\mathbf{4}$ is very compactly folded and the solvents fill the interstice between the chains of foldamers. Non-bonding hydrogens and solvent disorder have been omitted for clarity. Solvents are shown with space fill model and hydrogen bonds with turquoise.

In $5-\mathrm{CHCl}_{3}$ solvate, however, the overall conformation is less folded and bowl-like, as pyridine-2,6-dicarboxamide center P1 adopts an $\mathrm{S}$ fold. The bowl-shaped molecule is occupied by the ends of the neighboring foldamers and solvents. The mutually included assembly is further strengthened by intermolecular hydrogen bonds between a pair of foldamers occupying each other's cavities (H8N...O7; $R_{2}^{2}(14)$ motif). The hydrogen bonded pairs pack together in a $2 \mathrm{D}$ plane by $\pi$-stacking from the sides of the bowls. The gaps between the $2 \mathrm{D}$ planes are filled with disordered solvents.

\section{Structural comparison of the compounds}

The solid state structures of a series of foldamers 1-5 show that the hydrogen bonding and the folding patterns of the pyridine2,6-dicarboxamide units are reliably the same as observed with shorter oligoamide foldamers with only one pyridine-2,6dicarboxamide unit. ${ }^{18-20}$ The role of the spacer unit for the overall conformation becomes evident, when comparing foldamers 1-3. In foldamer 3 the rigidity of the spacer separates the pyridine centers to act like individual folding centers and hinder the formation of $S$ folds in the solid state. The position of individual centers in respect to relatively long, linear and rigid spacer may be either cis or trans, which induces either Z-shaped or bowl- shaped overall conformation, respectively. Both these conformations have an intrinsic niche or cavity for binding small guests, which is seen as solvent inclusion. In foldamers $\mathbf{1}$ and $\mathbf{2}$ the spacer is shorter and such individual behaviour of pyridine2,6-dicarboxamide units is not possible. In foldamer 1 the short phenyl spacer plays only a minor role in conformational preferences and four different crystal forms with two distinctly different overall conformers are hence likely caused by packing effects. The foldamer $\mathbf{2}$, on the other hand, has unsymmetrical spacer which exclusively favors @/S conformation in the solid state and the differences in overall conformations of different crystal structures are only minor.

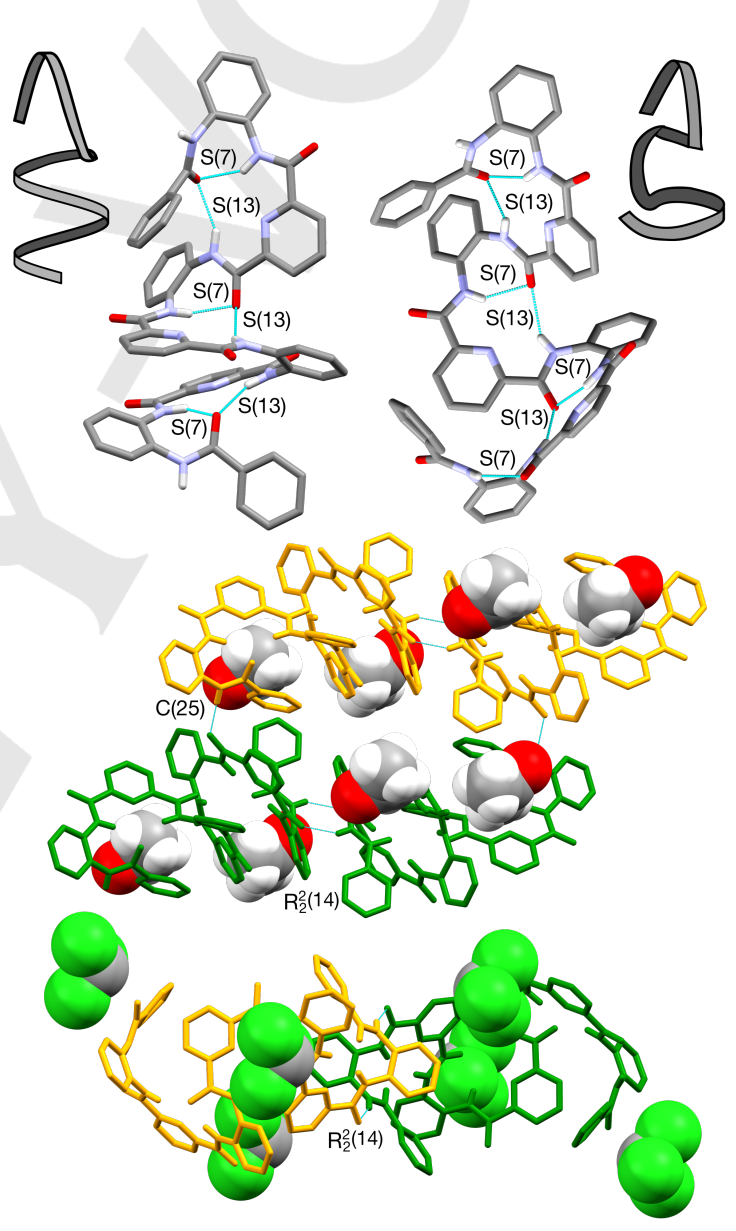

Figure 5 The conformations and packing of 5-Ac (top left and middle) and 5$\mathrm{CHCl}_{3}$ (top right and below). Aromatic hydrogens have been removed for clarity.

Changing the substitution positions of the central phenyl ring from ortho to meta in foldamer 4 increases the flexibility of the molecule which changes both the overall conformation and behaviour of pyridine-2,6-dicarboxamide units. More flexible linker part enables the formation and stabilization of $S$ folds by additional intramolecular hydrogen bonds. The overall conformation, however, is relatively compact. It is not possible to make any definite conclusions about the conformational stability 
and prevalence as only one crystal structure of foldamer $\mathbf{4}$ was obtained.

Introducing a third pyridine-2,6-dicarboxamide unit as a spacer in foldamer 5 changes the intramolecular hydrogen bonding significantly, which is seen as a reduction of number of hydrogen bonds from three to two in @ folded conformations of pyridine centers $\mathrm{P} 1$ and $\mathrm{P} 2$. The foldamer $\mathbf{5}$ can be seen as an extended version of foldamer $\mathbf{1}$ and they indeed share similar types of conformations (Figure 6). The foldamer 1 and 5 both have helical solvate structures and one more open structure. The difference between the two conformations observed is probably due to solvent interactions and packing.
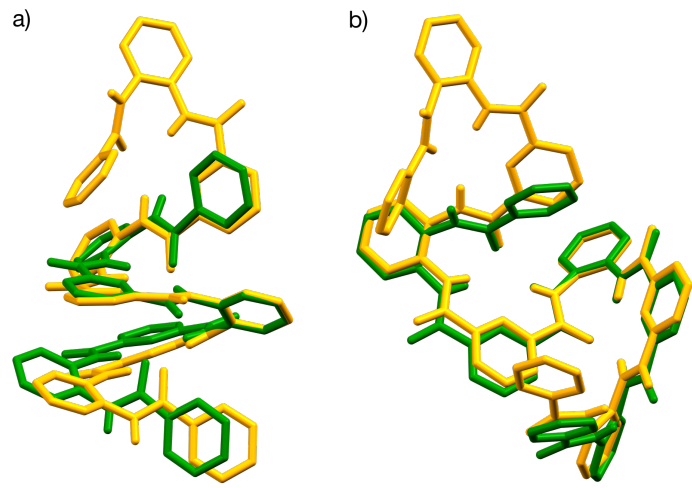

Figure 6 Comparison of the different conformations of foldamer 1 (green) and foldamer 5 (orange): a) comparison between the helical structures of 1-DMA$\mathrm{H}_{2} \mathrm{O}$ and 5-Ac. b) Comparison between the open structures of 1-DCM and 5$\mathrm{CHCl}_{3}$

\section{Solution state studies}

Solution state studies were performed for foldamers 1-5 to compare their solution state conformations with their solid state structures and to see, if the solid state motifs are observed in solution. To this end, a suite of $2 \mathrm{D}$ of homo- and heteronuclear correlation experiments was employed in addition to $1 \mathrm{D}{ }^{1} \mathrm{H}$ NMR and ${ }^{13} \mathrm{C}$ NMR experiments to yield a complete resonance assignment to all foldamers (see SI for details). 2D NOESY spectra were measured to obtain through space internuclear connectivities for conformational analysis. DMSO- $d_{6}$ was used as solvent in all samples. The 2D NOESY spectra show that the foldamers adopt folded conformations in the solution and in the case of foldamers 3-5 the data is fairly consistent with conformations seen in the crystal structures.

In the case of foldamer $\mathbf{1}$ the correlations show that the compound has a folded structure, but the correlations fit both solid state conformations equally well (see ESI Table S-3 for a detailed list of correlations). Two of the correlations, however, support the conclusion that the foldamer is folded also in solution (Figure 7a). The correlation a shows an interaction between the hydrogens of aromatic ring $A$ or $D$ and the amide $\mathrm{NH} 3$ or 4 . This correlation is possible in both @/@- and S/@conformations although based on the crystal structures 1-DMSO

and 1-DCM the correlation is stronger in the @/@-conformation. Another observation supporting the conclusion about a folded conformation is the correlation $b$, which is an interaction between the hydrogens of aromatic ring $\mathrm{B}$ or $\mathrm{C}$ and pyridine ring $\mathrm{P} 2$ or $\mathrm{P} 1$. This correlation is also possible in both @/@- and $\mathrm{S} / @$-conformations.

a) Foldamer 1 (1-DMSO)

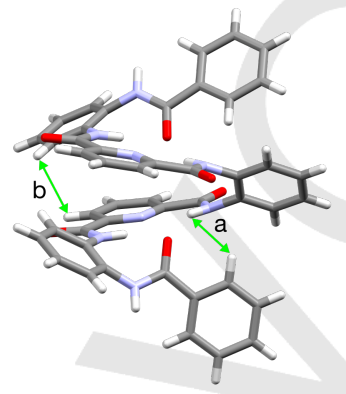

c) Foldamer 3 (3-DMSO)

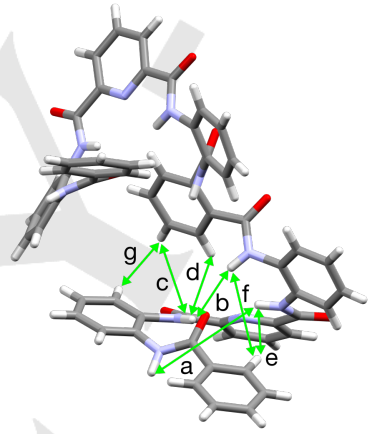

d) Foldamer 4 (4-EtOAc)

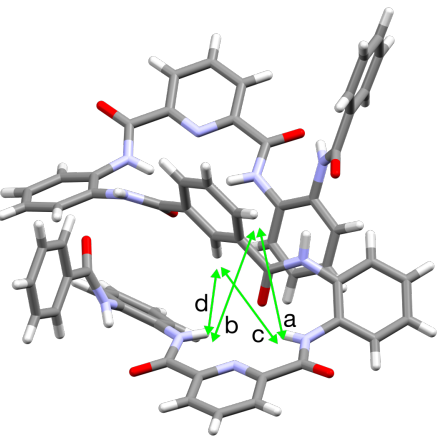

e) Foldamer 5 (5-acetone)

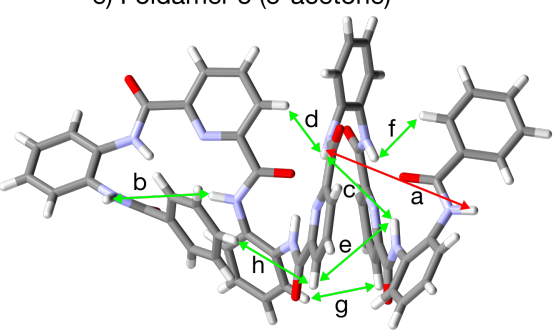

Figure 7 Selected NOE correlations of foldamers 1-5 shown in relation to relevant crystal structures. In symmetric foldamers only one set of correlations is shown. Correlations are marked with green if they are seen in the crystal structure and with red if they are not seen in the crystal structure

The solution state conformation of foldamer $\mathbf{2}$ is likely to deviate from the one observed in the solid state. The correlations of the one half of the foldamer match the solid state conformation, while the other end does not correspond to any of the interactions seen in the crystal structures. The deviating correlations a-e are all located at the pyridine center adopting the S-conformation in the crystal structure (Figure $7 \mathrm{~b}$; left side of the molecule, red lines). If the solution state conformation corresponded to the crystal structures, the correlation a should be found between $\mathrm{NH}_{1}$ and hydrogens on the opposite side of 
the aromatic ring $\mathrm{S} 2$ (para position to $\mathrm{NH} 4$ and $\mathrm{NH} 5$ ). Instead, the correlation is seen between $\mathrm{NH} 1$ and $\mathrm{S} 2$ hydrogens next to the amide groups. The same difference is observed with the correlation $c(\mathrm{NH} 2)$ with respect to the orientation of ring $\mathrm{S} 2$. Correlation $b$ between $\mathrm{NH} 2$ and aromatic ring $\mathrm{A}$ hydrogens is not possible in the crystalline state conformation, which indicates different orientation towards the end of the molecule compared with the crystal structures. Correlation $d$ between $\mathrm{NH} 2$ and aromatic ring $\mathrm{D}$ hydrogens and correlation e between $\mathrm{NH} 3$ and aromatic ring $\mathrm{A}$ hydrogens further confirm the tighter and more folded orientation of the molecule end in solution. Correlations $f-i$ ( $f=\mathrm{NH} 4-\mathrm{D} ; g=\mathrm{NH} 5-\mathrm{S} 1 ; h=\mathrm{NH} 5-\mathrm{D} ; i=\mathrm{NH} 6-\mathrm{S} 1)$ correspond well to the @-folded pyridine-2,6-dicarboxamide center of the crystal structures (Figure $7 \mathrm{~b}$; the right side of the molecule, green lines). These results indicate that the conformation of foldamer 2 in solution is probably @/@ instead of $@ / S$ that is exclusively seen in all crystal structures.

The NOE correlations a- $f$ of foldamer 3 agree well with the @ folds of the crystal structures (Figure 7c, Table 2). Additionally, a strong correlation $(g)$ between the aromatic hydrogens of rings $B$ and S2 suggests that the structure resembles the crystal structure 3-DMSO where the pyridine centers are in cis orientation with respect to the spacer. Given that DMSO was used as a solvent both in the solution and solid state studies, the observed similarities between the crystal structure and the NMR data are somewhat expected. Based on the roughly similar correlations of symmetrically equivalent bonds (see ESI Table S3 ), the conformation is nearly symmetrical in solution like in the solid state structure of 3 -DMSO. The correlation $f$, however, differs from the distances of the crystal structures. This can be explained by permanent hydrogen bonding to solvent DMSO in the crystal structure. Another minor difference between the solution and solid state structures is the stronger correlation a between $\mathrm{NH} 1$ and $\mathrm{NH} 3$ in solution. The NMR spectra also show some peaks, which could be identified as an additional solution state conformation. No clear NOE correlations, however, that could be used to determine the features of this conformation were identified.

The characterization of the solution state conformation of foldamer 4 was difficult because of many overlapping interactions and the presence of a second minor conformation in solution. Correlations a- $d$ from $\mathrm{NH} 2 / 7$ and $\mathrm{NH} 3 / 6$ protons to aromatic hydrogens still indicate a folded conformation (Figure $7 \mathrm{~d}$, Table S-3 in SI). Due to overlapping peaks of S2 and P1/P2 hydrogens and $S 2$ and $A / D$ hydrogens, however, it is not possible to unambiguously determine the conformations or how well they correspond to the solid state structure.

NOESY spectra of foldamer $\mathbf{5}$ show several correlations that confirm a folded solution state conformation (Figure 7e, Table S3 in ESI). Most of these correlations (b-f) are in agreement with both conformations observed in the crystal structures, while two correlations $(g=P 1-S 2$ and $h=P 3-D)$ narrow the conformation to resemble the@/@ structure, which is also more prevalent in the solid state. Correlation a, however, does not correspond to any of the interactions seen in the crystal structures. This suggests that although the solution state conformation resembles the helical @/@ conformation, it is still slightly different with respect to the orientation of the end of the molecule in the solid state. Alternatively, fast conformational exchange on the NMR timescale may take place between different structures.

\section{Conclusions}

Foldamers 1-5 with two or three pyridine-2,6-dicarboxamide centers and varying linker groups as their structural components show reliable folding patterns and stability of the desired oxyanion hole motif in respect to pyridine-2,6-dicarboxamide centers both in solution and in the solid state. Additionally, the number of pyridine-2,6-dicarboxamide centers can be multiplied without losing the essential structural features. The overall conformation of the foldamer varies depending on the linker unit. Foldamer $\mathbf{1}$ and foldamer $\mathbf{5}$, which is an extended version of $\mathbf{1}$, have similar ubiquitous helical conformations (@/@), but also alternative, more open structure in the solid state $(@ / S)$, probably caused by solvent effects during the crystallization. In the case of foldamer $\mathbf{1}$, no conclusive information about the solution conformation could be obtained, whereas in case foldamer $\mathbf{5}$ the prevalent conformation in solution appears to be @/@. The unsymmetrical linker unit of foldamer 2 directs the foldamers to have similar compact helical conformations in the solid state, whereas NOESY spectra indicate more folded @/@ type of conformer in solution. The flexibility of the metasubstituted linker group in foldamer $\mathbf{4}$ enables compact Sshaped folded conformation with additional intramolecular hydrogen bonds in contrast to the rigid ortho substituted spacer of foldamer 3 which enables the @-folded pyridine-2,6dicarboxamide units to orient in either trans or cis in relation to the center. The flexibility of foldamer $\mathbf{4}$ was also seen in solution, as no conclusive conformational information could be obtained and a possibility of alternative conformers was observed in NMR spectra.

Our future studies will orient toward utilizing extended foldamers as anion hosts utilizing their conformational predictability, and on the other hand, conformational adaptability without losing the binding site structure, which creates a suitable binding site for, for example, halogen anions. ${ }^{[2]}$ Additionally, anion binding capacity together with the resemblance to oxyanion hole motifs of enzymes provides excellent basis for future studies as enzyme-mimicking organocatalysts.

\section{Experimental Section}

\section{Materials and methods}

The synthesis and characterization details of foldamers 1-5 are presented in ESI. All starting materials were commercially available and used as such unless otherwise noted. Analytical grade solvents and Millipore water were used for crystallizations and slurries. NMR spectra were measured with Bruker Avance DPX250 MHz, Bruker Avance DRX $500 \mathrm{MHz}$ or with Bruker Avance III HD $800 \mathrm{MHz}$ spectrometer and the chemical shifts were calibrated to the residual proton and carbon resonance of the deuterated solvent. Melting points were measured in an 
Table 3. Crystal data and data collection parameters. The data of the isomorphous structures and the data of the structures 1-DMF- $\mathrm{H}_{2} \mathrm{O}$ and 1-MeOH are presented in ESI.

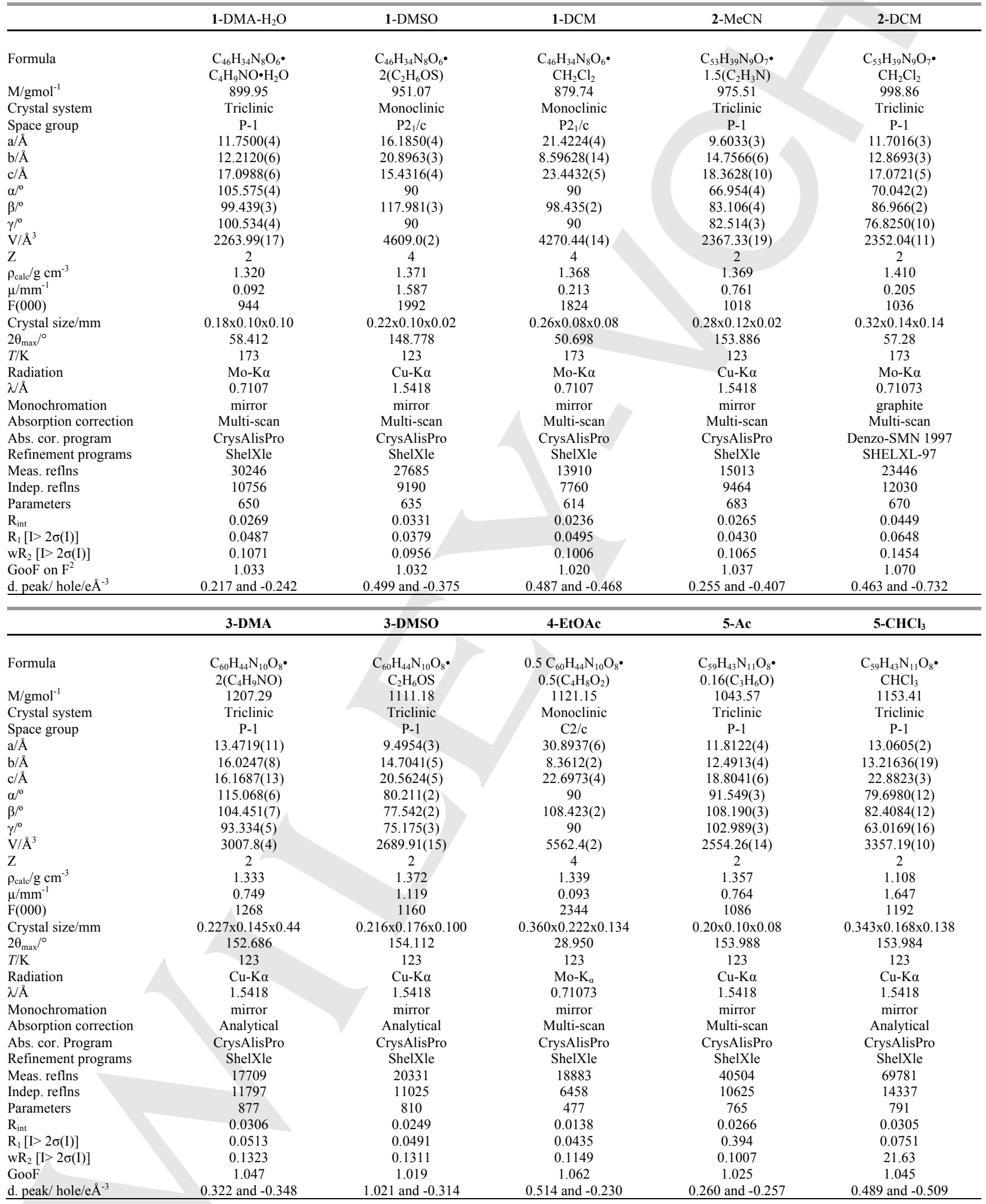


open capillary using a Stuart SMP30 melting point apparatus and are uncorrected. ESI-TOF mass spectra were measured with a LCT Micromass spectrometer.

\section{X-Ray Crystallography}

The crystal data and data collection parameters are presented in Table 3 and in $\mathrm{ESI}$ (isomorphous structures and the structures 1-DMF- $\mathrm{H}_{2} \mathrm{O}$ and 1-MeOH). General procedure for crystallization: $5-50 \mathrm{mg}$ of foldamers were dissolved in 0.1-6 $\mathrm{ml}$ of solvent. Heating and stirring were used to help the dissolving process. After the compounds had dissolved the solutions were allowed to evaporate at room temperature until the crystals formed. The details of crystallization and refinement are presented in ESI. Single crystal X-ray diffraction data of structures 1$\mathrm{MeCN}-\mathrm{H}_{2} \mathrm{O}$ and 2-DCM were measured with a Bruker Nonius KappaCCD diffractometer using a Bruker AXS APEX II CCD detector. Single crystal structures 1-MeOH, 1-DMSO, 2-MeCN, 2-EtOAc, 2-DMSO, 3-DMA, $3-$ DMSO, 5-DCM, 5-Ac, 5-DMF and 5- $\mathrm{CHCl}_{3}$ were measured with an Agilent Supernova Dualsource diffractometer and an Agilent Atlas CCD detector. Single crystal structures 1-DMF- $\mathrm{H}_{2} \mathrm{O}, 1-\mathrm{DMA}-\mathrm{H}_{2} \mathrm{O}, 1-\mathrm{DCM}$ and 4-EtOAc were measured with an Agilent Supernova diffractometer using an Agilent Eos CCD detector. All structures were solved with direct methods and refined using Fourier techniques. All non-hydrogen atoms were refined unisotropically, except for one acetonitrile in the structure 2$\mathrm{MeCN}$, which was refined isotropically due to disorder. The hydrogen atoms were placed in idealized positions except for the $\mathrm{N}-\mathrm{H}$ and $\mathrm{H}_{2} \mathrm{O}$ hydrogen atoms which were found from the electron density map. $\mathrm{N}-\mathrm{H}$ hydrogen $\mathrm{H} 5 \mathrm{NB}$ of structure 3-DMSO was placed in an ideal position, and included in the structure factor calculations. SQUEEZE was used on structure $5-\mathrm{CHCl}_{3}$ to remove severely disordered $\mathrm{CHCl}_{3}$ molecules that could not be modelled. Details of the crystal data and the refinement are presented Supporting information. Graph set symbols ${ }^{[25]}$ for hydrogen bonding were assigned and used to compare the hydrogen bonding between the different crystal structures. CCDC 1555244-1555260 contain the supplementary crystallographic data for this paper. These data can be obtained free of charge from The Cambridge Crystallographic Data Centre through www.ccdc.cam.ac.uk/data request/cif.

\section{Acknowledgements}

The financial support of Academy of Finland (proj. no. 257246 and 288235) is gratefully acknowledged. We thank B.Sc. Anniina Aho, M.Sc. Minna Kortelainen and M.Sc. Jussi Ollikka for the help in the synthesis, Spec. Lab. Technician Elina Hautakangas for the elemental analysis and Spec. Lab. Technician Esa Haapaniemi for the part of the NMR measurements.

Keywords: foldamers $\cdot$ hydrogen bonding $\cdot$ X-ray crystallography $\cdot$ NMR spectroscopy $\cdot$ molecular folding • supramolecular chemistry

[1] S. H. Gellman, Acc. Chem. Res. 1998, 31, 173-180.

[2] D. J. Hill, M. J. Mio, R. B. Prince, T. S. Hughes, J. S. Moore, Chem Rev. 2001, 101, 3893-4012.

[3] I. Huc, Eur. J. Org. Chem. 2004, 2004, 17-29.

[4] See for example: a) J. F. Galan, C. N. Tang, S. Chakrabarty, Z. Liu, G. Moyna, V. Pophristic, Phys. Chem. Chem. Phys. 2013, 15, 1188311892; b) Y. Yan, B. Qin, C. Ren, X. Chen, Y. K. Yip, R. Ye, D. Zhang H. Su, H. Zeng, J. Am. Chem. Soc. 2010, 132, 5869-5879; c) H. Jiang,
J. Léger, C. Dolain, P. Guionneau, I. Huc, Tetrahedron 2003, 59, 83658374; d) X. Hu, S. J. Dawson, Y. Nagaoka, A. Tanatani, I. Huc, J. Org. Chem. 2016, 81, 1137-1150.

[5] a) N. Delsuc, L. Poniman, J. Léger, I. Huc, Tetrahedron 2012, 68, 44644469; b) Z. Hu, H. Hu, C. Chen, J. Org. Chem. 2006, 71, 1131-1138; b) C. Dolain, J. Léger, N. Delsuc, H. Gornitzka, I. Huc, Proc. Nat. Acad. Sci. 2005, 102, 16146-16151.

[6] A. Suhonen, I. Morgan, E. Nauha, K. Helttunen, H. Tuononen and M. Nissinen, Cryst. Growth \& Des. 2015, 15, 2602-2608.

[7] H. Goto, H. Katagiri, Y. Furusho, E. Yashima, J. Am. Chem. Soc. 2006 128, 7176-7178.

[8] D. Sánchez-García, B. Kauffmann, T. Kawanami, H. Ihara, M. Takafuji, M. Delville, I. Huc, J. Am. Chem. Soc. 2009, 131, 8642-8648.

[9] E. Gillies, F. Deiss, C. Staedel, J. Schmitter, I. Huc, Angew. Chem. Int. Ed. 2007, 46, 4081-4084.

[10] a) M. Kudo, D. Carbajo López, V. Maurizot, H. Masu, A. Tanatani, I. Huc, Eur. J. Org. Chem. 2016, 2016, 2457-2466; b) N. Delsuc, F. Godde, B. Kauffmann, J. Léger, I. Huc, J. Am. Chem. Soc. 2007, 129, 11348-11349; c) J. Brüggemann, S. Bitter, S. Müller, W. Müller, U. Müller, N. Maier, W. Lindner, F. Vögtle, Angew. Chem. Int. Ed. 2007, 46, 254-259; d) C. A. Hunter, A. Spitaleri, S. Tomas, Chem. Commun. 2005, 3691-3693.

[11] B. Gong, Acc. Chem. Res. 2008, 41, 1376-1386.

[12] a) E. Kolomiets, V. Berl, J. Lehn, Chem. - Eur. J. 2007, 13, 5466-5479; b) M. Kudo, V. Maurizot, B. Kauffmann, A. Tanatani, I. Huc, J. Am. Chem. Soc. 2013, 135, 9628-9631.

[13] R. V. Nair, K. N. Vijayadas, A. Roy, G. J. Sanjayan, Eur. J. Org. Chem. 2014, 2014, 7763-7780

[14] A. Roy, P. Prabhakaran, P. K. Baruah, G. J. Sanjayan, Chem. Commun. 2011, 47, 11593-11611.

[15] C. Bao, Q. Gan, B. Kauffmann, H. Jiang, I. Huc, Chem. - Eur. J. 2009, $15,11530-11536$

[16] G. Lautrette, B. Wicher, B. Kauffmann, Y. Ferrand, I. Huc, J. Am. Chem. Soc. 2016, 138, 10314-10322.

[17] a) Y. Hamuro, S. J. Geib, A. D. Hamilton, Angew. Chem. Int. Ed. 1994, 33, 446-448; b) Y. Hamuro, S. J. Geib, A. D. Hamilton, J. Am. Chem Soc. 1996, 118, 7529-7541; c) Y. Hamuro, S. J. Geib, A. D. Hamilton, J. Am. Chem. Soc. 1997, 119, 10587-10593; d) V. Berl, I. Huc, R. G. Khoury, J. Lehn, Chem. -Eur. J. 2001, 7, 2798-2809; e) V. Berl, I. Huc, R. G. Khoury, J. Lehn, Chem. -Eur. J. 2001, 7, 2810-2820.

[18] A. Suhonen, E. Nauha, K. Salorinne, K. Helttunen, M. Nissinen, CrystEngComm 2012, 14, 7398-7407.

[19] M. Kortelainen, A. Suhonen, A. Hamza, I. Pápai, E. Nauha, S. Yliniemelä-Sipari, M. Nissinen, P. M. Pihko, Chem. Eur. J. 2015, 21 9493-9504

[20] A. Suhonen, M. Kortelainen, E. Nauha, S. Yliniemelä-Sipari, P. M. Pihko, M. Nissinen, CrystEngComm 2016, 18, 2005-2013.

[21] S-conformation is based on the hydrogen bonds between $C=O$ and pyridine-2,6-dicarboxamide NHs similarly to @ conformer, but the outmost $\mathrm{NH}$ group forms a hydrogen bond to a pyridine-2,6dicarboxamide $\mathrm{C}=\mathrm{O}$ (Scheme 1 ) or to another $\mathrm{C}=\mathrm{O}$ group of the foldamer or adjacent molecule, which turns the end of the molecule away from the pyridine core. Tighter and more folded @ conformation has the outer $\mathrm{N}-\mathrm{H}$ also turned towards the pyridine-2,6-dicarboxamide center with a hydrogen bond to carbonyl $\mathrm{C}=\mathrm{O}$ (S7 motif).

[22] P. M. Pihko, S. Rapakko, R. K. Wierenga in Hydrogen Bonding in Organic Synthesis (Ed.: P. M. Pinko), Wiley-VCH, Weinheim, 2009, pp. 43-71.

[23] a) K. Mitsui, S. A. Hyatt, D. A. Turner, C. M. Hadad, J. R. Parquette, Chem. Commun. 2009, 3261-3263; b) N. T. Salzameda, D. A. Lightner, Monatsh. Chem. 2007, 138, 237-244.

[24] For anion binding properties of small analogues see: K. Helttunen, R. Annala, A. Suhonen, E. Nauha, J. Linnanto, M. Nissinen, CrystEngComm, 2017, DOI: 10.1039/C7CE01109A. 
[25] a) M.C. Etter, J. C. MacDonald, Acta Crystallogr., Sect. B: Struct. Sci., 1990, 46, 256-262; b) J. Bernstein, R. E. Davis, L. Shimoni, N.-L. 


\section{Entry for the Table of Contents}

Layout 1:

\section{FULL PAPER}

Predictability and persistence of folding: Aromatic oligoamide foldamers fold into helices and more open, folded conformations depending on the identity of central linker and solvent conditions. Pyridine-2,6-dicarboxamide units add the predictability of the conformation and lead to good persistence and comparability of conformation in solution and in the solid state.
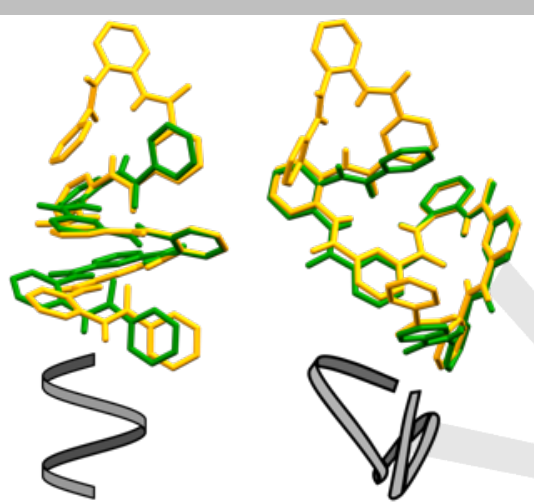

Riia Annala, Aku Suhonen, Heikki Laakkonen, Perttu Permi, Maija Nissinen ${ }^{*}$

Page No. - Page No.

Structural tuning and

conformational predictability of aromatic oligoamide foldamers 